\title{
Translational research in Radio-Oncology: how to integrate it into clinical orthodoxy?
}

In a recent past translational research emerged as one of the driving forces in Medicine and more particularly in Oncology. Actually radiation science and its clinical applications turns out to be one of the most fertile grounds of cancer research for successful translational studies, as recently demonstrated by the Second Edition of the International Conference on Translational Research and Pre-Clinical Strategies in Radiation Oncology (ICTR2003), hold in 2003, in Lugano, Switzerland.

To understand this successful evolution within the Radio-Oncology community, we need first to spend some considerations on semantics. Is there indeed a satisfactory definition provided so far for translational research? Probably not, for most of them focus too much on transfer of information from an experimental environment to a clinical setting. Actually translational research represents a very broad collection of knowledge and expertise, which embrace sequential concepts, distinct in time and space.

First translational research follows, throughout the development of a therapeutic strategy for a given disease, a timeline originating from fundamental research, which actually represents the "feeding" process for all further steps of research, encompassing the whole chain of developmental works including applied research, pre-clinical strategies, clinical validation of treatment safety and efficacy, and leading at the end of the process to the routine application of the novel drug or technique as standard treatment for a given disease. When it is successful, this process can require up to 15-20 years before its happy end.

It is also of fundamental importance to acknowledge in translational research the need for mechanisms of feed-back, namely from the clinic to the lab, when crucial information retrieved from tumor and normal tissue response to treatment, imposes a critical appraisal of both experimental data and clinical delivery modalities, as is was the case for instance for altered fractionation (minimum time interval between irradiation daily sessions to ensure sufficient normal tissue repair within the framework of accelerated regimes of radiotherapy) or with regimes combining chemo- and radiotherapy (unacceptable enhancement of toxicity in normal tissues such as high grade mucositis).
The notion of space or more exactly of environment dimensions is also of critical importance to understand the algorithms used in translational studies. Two years ago Coleman ${ }^{1}$ very nicely documented this concept by stratifying the targets of translational research in radiation science according to: the macro-environment, corresponding to the subject's body and therefore to normal tissue calling for studies on therapeutic index optimization; the normo-environment referring to tumor and tissue blood supply and subject to predictive assays; the micro-environment related to tissular and cellular pattern and investigated by cell biology, and finally the nano and pico-environment corresponding to treatment effects on cytosol and nucleus elements, implying approaches based on bio-molecular targeting.

But to be successful, translational research has to pack up concerted actions in the framework of another concept, which is that of a reinforced integration of Radio-Oncology disciplines (namely imaging, biology, radiation physics and clinics) into the developmental work in our laboratories.

Without this further integration, treatment optimization would face serious limitations, especially in our search for modalities offering more favorable therapeutic indexes, in other words a better discriminatory effect between normal tissue and tumor cells. As a matter of fact nano- and pico-environments are the cornerstone of this translational research, and both functional imaging and molecular-targeted therapies are now at the center of a large number of prospective investigations, but we would make a mistake if we should consider this pathway as the only one leading to a significant increase in disease local and regional control. Radiation physics must indeed be part of this optimization process since it is on the verge to offer, thanks to intensity-modulated planning and delivery processes and hadrontherapy, very powerful tools that will allow a significant increase in tumor dose and a better sparing of surrounding normal tissues ${ }^{2}$. The main challenge remains intrinsic radio-resistance in tumors with very radio-resistant genotypes, within given histological types, for which no satisfactory solution is at our doors. Here we'll have to develop inventive genetic manipulations in order to alter DNA 
repair mechanisms. Some results recently obtained in fundamental research undoubtedly constitute a strong impetus to strengthen this field of translational studies.

We should nevertheless never forget that, in translational research, the key of success also lies in the lessons we have drawn and will have to draw from our failures, which have been shown to be strong incentives for more concerted actions among our laboratories and a more intensive dialog between clinicians and scientists, a dialog physicians have ignored for a too long time.

\section{References}

1. Coleman CN. International conference on translational research and preclinical strategies in radio-oncology (ICTR): conference summary. Int J Rad Oncol Biol Phys 2001;49: 301-9.

2. Bernier J, on behalf of the ICTR 2000 Organising Committee. ICTR 2000: individualizing cancer treatment. Int J Rad Oncol Biol Phys 2001;49:299.

J. Bernier Chairman, Radio-Oncology Department. Oncology Institute of Southern Switzerland. Bellinzona. Switzerland. 